\title{
Convergence of Simulation-Based Policy Iteration *
}

\author{
William L. Cooper \\ Department of Mechanical Engineering \\ University of Minnesota, 111 Church Street S.E., Minneapolis, MN 55455 \\ billcoop@me.umn.edu(612)624-4322 \\ Shane G. Henderson \\ School of Operations Research and Industrial Engineering \\ 230 Rhodes Hall, Cornell University, Ithaca, NY 14853 \\ shane@orie.cornell.edu (607) 255-9126 \\ Mark E. Lewis ${ }^{\dagger}$ \\ Department of Industrial and Operations Engineering \\ University of Michigan, 1205 Beal Avenue, Ann Arbor, MI 48109-2117 \\ melewis@engin.umich.edu(734) 763-0519
}

January 16,2002

\begin{abstract}
Simulation-based policy iteration (SBPI) is a modifcation of the policy iteration algorithm for computing optimal policies for Markov decision processes. At each iteration, rather than solving the average evaluation equations, SBPI employs simulation to estimate a solution to these equations. For unichain average-reward Markov decision processes with £nite state and action spaces, we provide easily-verifable conditions that ensure that simulation-based policy iteration almost-surely eventually never leaves the set of optimal decision rules. We analyze three simulation estimators for solutions to the average evaluation equations. Using our general results, we derive simple conditions on the simulation runlengths that guarantee the almost-sure converge of the algorithm.
\end{abstract}

${ }^{*}$ MSC Subject Classifcations: primary-90C40: Markov and semi-Markov decision processes, secondary-68U20: Simulation

${ }^{\dagger}$ Corresponding author. 


\section{Introduction}

The policy iteration (PI) algorithm is a method for computing optimal policies in Markov decision processes (MDPs). In essence the algorithm consists of a policy evaluation step in which the value (the precise meaning of value depends upon the choice of optimality criterion) of the current policy is computed, and a policy improvement step where, if possible, the current policy is improved upon. These two steps are repeated iteratively until some stopping requirements are met. In this paper, we focus on the average-reward optimality criterion for unichain MDPs with £nite state and action spaces. For such MDPs the PI algorithm is known to converge to an optimal policy in a £nite number of iterations.

The evaluation step of policy iteration consists of solving a set of linear equations called the average evaluation equations (AEE) or Poisson's equation. Using the solution to the AEE, the improvement step then employs a one-step analysis to decide if the current policy can be improved. For MDPs with large state spaces, the linear systems that must be solved in the evaluation step can be prohibitively large, thereby rendering the PI algorithm impractical. This phenomenon is, of course, the well-known "curse of dimensionality," which causes severe dif£culties for this (and all other) MDP solution procedures.

In this paper we analyze simulation-based policy iteration (SBPI), a modifcation of the policy iteration algorithm described in $[2,3,5,6,7]$. Rather than exactly solving the AEE in the policy evaluation step, SBPI estimates solutions of the AEE via simulation. It then uses these estimates as a proxy for the exact AEE solution in the policy improvement step. Note that this procedure does not require the solution of the

large linear system. Provided that SBPI employs such "reasonable" estimators, the mean squared error of the estimates of the AEE solution will converge to zero as the runlength of each simulation grows to infnity, Since "regular" PI converges to an optimal policy, one might then conjecture that SBPI should converge almost-surely (or in probability) to an optimal policy, so long as the runlengths grow to infnity. This is, in fact, not the case; we present a counter-example to this effect.

In light of this observation, it is natural to ask what conditions do ensure the almost-sure convergence of SBPI. Several earlier papers have given partial answers to this question. For instance, Cao [6] shows that if simulation estimates are "close enough" to a solution of the AEE, then SBPI will stop at an optimal policy. In addition, he notes that as the runlengths of the simulations grow to infnity, the simulation estimates converge with probability one to a solution of the AEE. However, he does not provide verifable conditions on the runlengths that ensure that estimates are indeed accurate enough. As the above-mentioned example 
will show, allowing the runlengths to grow to in£nity does not suffce for almost-sure convergence of the algorithm.

We present easily-verifable conditions that guarantee the almost-sure convergence of the simulationbased algorithm. Thus, the Erst contribution of this paper is to close the gap in the literature left by the interesting papers of Cao [5, 6, 7] and Cao and Chen [8]. Our second contribution is the presentation of explicit convergence conditions related to three speci£c estimators each based upon probabilistic interpretations of the AEE. In particular, we describe how to estimate two possible solutions; the bias (one estimator) and the relative value function (two different estimators). We apply our results to obtain explicit conditions on the runlengths (or the appropriate analog) of each estimator to guarantee almost-sure convergence of the algorithm. For two of the three estimators, our results hold under the assumption that the MDP in question is unichain. For the third, we require a slightly stronger condition - namely that all stationary policies yield a common single recurrent class with no transient states. Cao [6] apparently does not distinguish between unichain and the stronger condition.

Other methods similar to SBPI include the adaptive critic algorithm $[1,11]$ and the modifed policy iteration algorithm [17, Section 8.7.1]. In the prior, approximate solutions in both the policy evaluation and policy improvement steps are obtained using simulation and simple recursions, while in the latter approximate solutions in the policy evaluation step are obtained using value iteration. Like modifed policy iteration, we obtain an approximate solution in the policy evaluation step and solve the policy improvement step exactly. Another approach to solving MDPs is through the use of Q-learning algorithms [3, 21, 22]. This approach is applicable when one does not have explicit knowledge of the transition matrix, and may be viewed as a simulation-based variant of the value iteration method for solving MDPs.

The rest of the paper is organized as follows. Section 2 gathers useful defnitions and results from MDP theory, thereby setting the framework for the remainder of the paper. Section 3 describes a simple example for which SBPI does not converge, even though runlengths grow to in£nity. Section 4 provides a general result that guarantees the almost-sure convergence of SBPI. Section 5 shows how to apply the general result to several specifc estimators to yield explicit conditions that ensure convergence. Section 6 provides a brief summary and conclusions. 


\section{Markov Decision Process Theory}

In this section we discuss an average reward criterion Markov Decision Process and introduce the policy iteration algorithm. Our notation closely follows that of Puterman [17]. Assume that the state space $\mathbb{X}$ and the action space $\mathbb{A}$ are £nite. That is to say that when in state $x \in \mathbb{X}$ there is a fnite set of actions $A_{x}$ $\left(\mathbb{A}=\cup_{x \in \mathbb{X}} A_{x}\right)$ from which a decision-maker can choose. Once the action $a$ is chosen, a reward $r(x, a)<\infty$ is accrued, the process moves to state $y$ with probability $p(y \mid x, a)$ and the process continues.

A deterministic decision rule $d$ is a map from $\mathbb{X}$ to $\mathbb{A}$ such that when in state $x$, the action $d(x)$ is used. A deterministic, Markovian policy $\psi$ is a sequence of decision rules that describes what decisions will be made for every decision epoch. That is to say that $\psi$ is of the form $\left\{d_{0}, d_{1}, d_{2}, \ldots\right\}$. We are interested in the class of stationary, deterministic policies which use the same decision rule for all decision epochs. A policy in this class is of the form $\{d, d, \ldots\}$ and is denoted $d^{\infty}$. Let $P_{d}$ be the one step transition matrix whose $x y$ element is $p(y \mid x, d(x))$, and let $\Psi$ denote the set of all non-anticipatory policies. We are now ready to de£ne the gain and the bias of a policy $\psi$. Let $X_{k}$ be the state of the system at stage $k$ and $d_{k}$ the decision rule at stage $k$ under a particular policy $\psi$. The $k$-stage expected reward of the policy $\psi$ given that the initial state is $x$ is given by

$$
J_{\psi}^{k}(x) \equiv E_{\psi}^{x}\left[\sum_{n=0}^{k-1} r\left(X_{n}, d_{n}\left(X_{n}\right)\right)\right]
$$

where $E_{\psi}^{x}$ denotes expectation with respect to the probability measure determined by the initial state $x$ and the policy $\psi$.

The long-run average reward or gain of a policy $\psi$ given that the system started in state $x$ is given by

$$
g_{\psi}(x)=\liminf _{k \rightarrow \infty} J_{\psi}^{k}(x) / k
$$

The optimal expected average reward is $g^{*}(x)=\sup _{\psi \in \Psi} g_{\psi}(x)$. A policy $\psi^{*}$ is called long-run average or gain optimal if $g_{\psi}(x)=g^{*}(x)$ for all $x \in \mathbb{X}$. If the Markov chain generated by a stationary, deterministic policy $\psi$ is aperiodic, the bias of $\psi$ given that the system started in state $x$ is de£ned to be

$$
h_{\psi}(x)=\sum_{n=0}^{\infty} E_{\psi}^{x}\left[r\left(X_{n}, \psi\left(X_{n}\right)\right)-g_{\psi}\left(X_{n}\right)\right] .
$$


A slightly different defnition of bias is required for periodic chains; however, we will not require the added level of generality in this paper. For MDPs with multiple gain-optimal policies, the bias provides a natural criterion for selecting among them. For more detailed studies of the bias (and bias optimality) see Lewis and Puterman $[13,12]$.

Let the set of all deterministic decision rules be denoted $D$ and the set of deterministic stationary policies be denoted $D^{\infty}$. In the coming sections we focus on policies in $D^{\infty}$. So, in the interest of notational simplicity, we will use the notation $d$ to mean both the policy $d^{\infty}=\{d, d, \ldots\}$ and the decision rule $d$; the precise meaning should be clear from the context. We will use the notation $d^{\infty}$ when extra clarity is needed.

Before proceeding, we need a bit more terminology. Given a real-valued function $f$ defned on $\mathbb{X}$, a decision rule $d^{\prime} \in D$ is an element of $\arg \max _{d \in D}\left\{r_{d}+P_{d} f\right\}$ if

$$
d^{\prime}(x) \in \arg \max _{a \in A_{x}}\left\{r(x, a)+\sum_{y \in \mathbb{X}} p(y \mid x, a) f(y)\right\} \quad \text { for each } x \in \mathbb{X} .
$$

Unless otherwise stated, for the remainder of the paper we assume that the Markov Decision processes under consideration are unichain. That is to say that all stationary, deterministic policies induce Markov chains that have one recurrent class and, perhaps some transient states. Thus

1. The gain of any stationary, deterministic policy $d^{\infty} \in D^{\infty}$ is a constant vector which we express as $g_{d} 1$.

2. If $(g, h)$ satisfes

$$
h=r_{d}-g 1+P_{d} h
$$

then $g=g_{d}$ and $h$ is unique up to a constant. The set of equations (2.3) are referred to as the average evaluation equations (AEE).

3. The pair $\left(g_{d}, h_{d}\right)$ is the only solution of (2.3) that also satis£es the additional condition $P_{d}^{*} h=0$, where $P_{d}^{*}$ represents the stationary distribution of the Markov chain generated by $d^{\infty}$.

4. The unique solution to the AEE that also satisfes $h(\alpha)=0$ for a particular state $\alpha$ is called the relative value function of $d$ with reference state $\alpha$.

Thus, for a £xed policy, if we can estimate the gain and either the bias or a relative value function, we have an estimate of the solution of the AEE. 
It is well-known that if $(g, h)$ satis£es

$$
h=\max _{d \in D}\left\{r_{d}-g 1+P_{d} h\right\}
$$

then $g=g^{*}$ and $h$ is unique up to a constant. The set of equations (2.4) are referred to as the average optimality equations (AOE). Let $D^{*}$ be the set of decision rules that achieve the maximum in (2.4). That is, if $(g, h)$ is a solution to the AOE, then

$$
D^{*} \equiv \arg \max _{d \in D}\left\{r_{d}+P_{d} h\right\}
$$

We refer to (2.5) as the average optimality selection equations. Theorem 8.4.2 of Puterman [17] guarantees that $D^{*} \neq \varnothing$ and for any $d^{*} \in D^{*}$, the stationary policy $\left(d^{*}\right)^{\infty}$ is gain optimal.

We conclude this section with a brief discussion of the policy iteration algorithm and the simulationbased policy iteration algorithm. The PI algorithm was originally discussed by Howard [10]. The reader is also referred to Chapter 8 of Puterman [17]. The SBPI algorithm was suggested by Bertsekas [2] and discussed further by Cao [5, 6, 7]. However, we emphasize that neither of the aforementioned authors provide veri£able convergence conditions for the algorithm.

\section{Policy Iteration Algorithm}

- Step (i). Initialization. Select a decision rule $d_{0} \in D$, set $j=0$.

- Step (ii). Policy Evaluation. Obtain a solution $\left(g_{d_{j}}, h_{j}\right)$ to the AEE for the decision rule $d_{j}$.

- Step (iii). Policy Improvement. Choose $d_{j+1}$ that satis£es

$$
d_{j+1}(x) \in \arg \max _{a \in A_{x}}\left\{r(x, a)+\sum_{y \in \mathbb{X}} p(y \mid x, a) h_{j}(y)\right\} \quad \text { for each } x \in \mathbb{X}
$$

setting $d_{j+1}(x)=d_{j}(x)$ whenever possible.

- Step (iv). Iteration. If $d_{j+1}=d_{j}$, then stop. Otherwise, let $j=j+1$ and return to Step (ii).

The key convergence results for the PI algorithm for unichain MDPs with £nite state and action spaces are collected in [17, Section 8.6]. There, it is demonstrated that at each iteration of the PI algorithm, there is a strict increase in the gain, and if not, then there is a strict increase in the bias. If neither occurs, then 
the decision rule must be the same as in the previous iteration. In this case we have an element of $D^{*}$, and hence a gain optimal policy. Since there are only £nitely many stationary, deterministic policies when the state and action spaces are £nite, the algorithm is guaranteed to terminate in £nitely many steps with the desired average reward optimal policy.

To specify the SBPI algorithm we replace the second step of the PI algorithm with an estimate $\left(g_{d_{j}}^{n_{j}}, h_{d_{j}}^{n_{j}}\right)$ of a solution to the AEE, where the sequence $\left\{n_{j}\right\}$ are pre-specifed parameters of the estimates. Note that in most cases $n_{j}$ will simply be the number of simulation runs used to obtain the estimate at iteration $j$, but we allow them to be more general. A second difference is that at the iteration step we do not specify a stopping criterion. The SBPI algorithm follows.

\section{Simulation-Based Policy Iteration Algorithm}

- Step (i). Initialization. Choose a sequence $\left\{n_{j}: j \geq 0\right\}$, and a decision rule $d_{0}$. Let $j=0$.

- Step (ii). Policy Evaluation Approximation. Obtain an estimate of the solution to the AEE $\left(g_{d_{j}}^{n_{j}}, h_{d_{j}}^{n_{j}}\right)$ for the decision rule $d_{j}$.

- Step (iii). Policy Improvement. Using our current estimate, $£$ nd a decision rule $d_{j+1}$ that satis£es

$$
d_{j+1}(x) \in \arg \max _{a \in A_{x}}\left\{r(x, a)+\sum_{y \in \mathbb{X}} p(y \mid x, a) h_{d_{j}}^{n_{j}}(y)\right\} \quad \text { for each } x \in \mathbb{X},
$$

setting $d_{j+1}(x)=d_{j}(x)$ whenever possible.

- Step (iv). Iteration. Let $j=j+1$ and return to Step (ii).

Since we are only estimating solutions to the AEE, the fact that a policy repeats does not guarantee optimality. One way to incorporate a stopping rule is to perform a step of standard policy iteration whenever we think that we possess an optimal decision rule. If after this iteration, we have the same decision rule, then we have arrived at an optimal policy. Alternatively, we could stop if the SBPI algorithm repeats the same policy for "enough" consecutive iterations. We leave the analysis of stopping rules as a subject for future research.

Note also that in the policy evaluation approximation step we estimate the average cost, yet in the policy improvement step we do not require this estimate. Indeed, all that is required for the policy improvement 
step is $h_{d_{j}}^{n_{j}}$. In light of the fact that no stopping rule is speci£ed, we feel that having a running gain estimate is an "added bonus". Since not much extra work is required to obtain the estimate, it is included in the algorithm. Finally, the condition that we set $d_{j+1}(x)=d_{j}(x)$ whenever possible is not needed for the primary results below. We could allow ties to be broken in any arbitrary manner, so long as the tie-breaking mechanism does not depend upon the past (or future) evolution of the algorithm.

\section{A Counterexample}

In this section we show by example that simulation-based policy iteration need not converge almost-surely to the set of optimal policies, even when the number of simulation replications at each iteration grows to infnity. In subsequent sections, we present conditions that do guarantee such convergence.

Consider the following simple example with three states, labelled 1, 2, and 3. We obtain a single-period reward of 0 whenever we are in state 1, and a single-period reward of 1 whenever we are in state 2 or state 3. When in state 1 , there are two possible actions, $\alpha$ and $\beta$; so $A_{1}=\{\alpha, \beta\}$. Choosing action $\alpha$ causes us to jump to state 2 with probability 1 , whereas choosing action $\beta$ causes us to jump to state 3 with probability 1. We assume that the action sets $A_{2}$ and $A_{3}$ are singletons; i.e., we have no decision when in states 2 and 3. When in state 2 , we return to state 2 with probability $p$ and jump to state 1 with probability $1-p$. Similarly, when in state 3 , we return to state 3 with probability $1-p$ and jump to state 1 with probability $p$. Suppose that $p \in(1 / 2,1)$.

For the example described above, there are only two decision rules to consider. In a slight abuse of notation, we shall call these rules $\alpha$ and $\beta$, corresponding to the action chosen in state 1. A simple check shows that the gain of $\alpha$ is $g_{\alpha}=1 /(2-p)$, and that the gain of $\beta$ is $g_{\beta}=1 /(1+p)$. Since we have assumed that $p \in(1 / 2,1)$, it follows that the optimal decision rule is $\alpha$.

It is easy to show that a solution to the AEE is given by $\left(g_{d}, v_{d}\right)$, where

$$
v_{d}(x)=E_{d}^{x} \sum_{n=0}^{\tau_{1}-1}\left[r\left(X_{n}, d\left(X_{n}\right)\right)-g_{d}\left(X_{n}\right)\right]
$$


and $\tau_{1}=\inf \left\{n \geq 0: X_{n}=1\right\}$. Computing this function for each of our policies gives us

$$
v_{\alpha}=\left(\begin{array}{c}
0 \\
\frac{1}{2-p} \\
\frac{1-p}{p(2-p)}
\end{array}\right) \text { and } v_{\beta}=\left(\begin{array}{c}
0 \\
\frac{p}{(1-p)(1+p)} \\
\frac{1}{1+p}
\end{array}\right)
$$

Since $v_{\alpha}(1)=v_{\beta}(1)=0, v_{d}$ is a relative value of $d$ with reference state 1 . Direct computations show that $v_{\alpha}(2)>v_{\alpha}(3)$ and $v_{\beta}(2)>v_{\beta}(3)$, so that the policy improvement step of deterministic policy iteration selects $\alpha$ when the "current" policy is either $\alpha$ and $\beta$; i.e., $\arg \max \left\{r_{d}+P_{d} v_{\alpha}\right\}=\arg \max \left\{r_{d}+P_{d} v_{\beta}\right\}=\alpha$.

Next we describe a particular implementation of simulation-based policy iteration that employs simulation estimates of $v_{\alpha}$ and $v_{\beta}$. To make things as simple as possible, we will use the exact values of $g_{\alpha}$ and $g_{\beta}$ in constructing our estimates. (We could also estimate the gain using the methods described in Section 5.2. If we did this, we could ensure that the estimate was close enough to the actual gain that the effects described below would still prevail. Since this would merely make things more complicated, but not add any additional insight, we shall simply use the actual gain for this example.) Since we know $v_{d}(1)=0$ without doing any computations (because $v_{d}$ is the relative value function with reference state 1 ), we need not estimate its value at state 1 . Note also that $v_{\alpha}(1)$ and $v_{\beta}(1)$ are both multiplied by 0 in the policy-improvement step. Hence, we need only estimate the values of $v_{d}(2)$ and $v_{d}(3)$.

To generate a simulation estimate for both $v_{\alpha}(2)$ and $v_{\alpha}(3)$, we employ a single draw from the canonical space of in£nite sequences of uniform- $(0,1)$ random variables. Denote a generic element from this space by $\mathbf{u}=\left\{u_{n}: n=0,1,2, \ldots\right\}$. We construct the estimates $\hat{v}_{\alpha}(2)$ and $\hat{v}_{\alpha}(3)$ by using the entries of $\mathbf{u}$ to generate transitions of the Markov chain induced by policy $\alpha$.

Let $T: \mathbb{X} \times(0,1) \rightarrow \mathbb{X}$ be the transition function that maps the current state of the Markov chain and a single uniform- $(0,1)$ to the next state of the Markov chain. We take

$$
\begin{aligned}
& T(2, u)= \begin{cases}2 & \text { for } u \in(0, p) \\
1 & \text { for } u \in[p, 1),\end{cases} \\
& T(3, u)= \begin{cases}1 & \text { for } u \in(0, p) \\
3 & \text { for } u \in[p, 1) .\end{cases}
\end{aligned}
$$

We will not need to generate transitions from state 1 , so we do not discuss this. For $x=2,3$, let $\left\{X_{n}^{x}\right\}$ denote the chain started in state $x$ that uses $\mathbf{u}$ and $T$ to make state transitions. It is crucial to note that $\left\{X_{n}^{2}\right\}$ 
and $\left\{X_{n}^{3}\right\}$ evolve according to the same $\mathbf{u}$. Our simulation estimators are

$$
\hat{v}_{\alpha}(x)=\sum_{n=0}^{\tau_{1}^{x}-1}\left(r\left(X_{n}^{x}\right)-g_{\alpha}\right)=\sum_{n=0}^{\tau_{1}^{x}-1}\left(1-\frac{1}{2-p}\right)=\sum_{n=0}^{\tau_{1}^{x}-1} \frac{1-p}{2-p} \quad \text { for } x=2,3
$$

where $\tau_{1}^{x}$ is hitting time of state 1 for the chain $\left\{X_{n}^{x}\right\}$. It is easy to check that for $x=2,3, \hat{v}_{\alpha}(x)$ is an unbiased estimator for $v_{\alpha}(x)$. In light of our joint construction, we see that

$$
\begin{aligned}
& P\left(\hat{v}_{\alpha}(2)=m \times \frac{1-p}{2-p}, \hat{v}_{\alpha}(3)=\frac{1-p}{2-p}\right)=p^{m-1}(1-p) \quad m=2,3, \ldots \\
& P\left(\hat{v}_{\alpha}(2)=\frac{1-p}{2-p}, \hat{v}_{\alpha}(3)=m \times \frac{1-p}{2-p}\right)=p(1-p)^{m-1} \quad m=2,3, \ldots
\end{aligned}
$$

Note that by virtue of our construction, exactly one of the two versions immediately hits state 1 . Using $T$, we can also generate analogous estimators for decision rule $\beta$. Mimicking the development above, we get

$$
\begin{aligned}
& P\left(\hat{v}_{\beta}(2)=m \times \frac{p}{1+p}, \hat{v}_{\beta}(3)=\frac{p}{1+p}\right)=p^{m-1}(1-p) \quad m=2,3, \ldots \\
& P\left(\hat{v}_{\beta}(2)=\frac{p}{1+p}, \hat{v}_{\beta}(3)=m \times \frac{p}{1+p}\right)=p(1-p)^{m-1} \quad m=2,3, \ldots
\end{aligned}
$$

By (3.3)-(3.6) it follows that

$$
P\left(\hat{v}_{\alpha}(3)>\hat{v}_{\alpha}(2)\right)=P\left(\hat{v}_{\beta}(3)>\hat{v}_{\beta}(2)\right)=1-p .
$$

Now, consider a simulation-based policy improvement step based upon the average $\hat{v}_{d}^{n}$ of $n$ independent replications of $\hat{v}_{d}$. Elementary calculations show that if $\hat{v}_{d}^{n}(3)>\hat{v}_{d}^{n}(2)$, then simulation-based policy improvement will choose policy $\beta$ (which is not the choice that would have been made by deterministic policy improvement). A suf£cient condition for $\hat{v}_{d}^{n}(3)>\hat{v}_{d}^{n}(2)$ is that each individual replication satis£es $\hat{v}_{d}(3)>\hat{v}_{d}(2)$. Therefore by (3.7), when using $n$ independent replicates to perform policy improvement, the probability of error is at least $\epsilon_{n} \equiv(1-p)^{n}$, and consequently, the probability of making the correct choice is at most $1-\epsilon_{n}$.

The above construction provides a concrete example that allows us to see explicitly how incorrect choices can be made in the policy improvement step. To see what ramifcations this has for the possible 
convergence of SBPI, £x $\epsilon \in(0,1)$, and let $\xi(n)=\min \left\{i:\left(1-\epsilon_{n}\right)^{i}<\epsilon\right\}$. Suppose now that we implement SBPI using the above constructions with the number of replications at iteration $j$ given by

$$
n_{j}=n \text { for } j \in[\theta(n-1)+1, \theta(n)]
$$

where $\theta(n)=\sum_{i=1}^{n} \xi(i)$. Note that $\xi(n)$ is the number of successive times that $n$ is used as the number of replications. By the defnitions of $\xi(n)$ and $\epsilon_{n}$, we see that $\xi(n) \rightarrow \infty$ as $n \rightarrow \infty$. Furthermore, $n_{j} \rightarrow \infty$ as $j \rightarrow \infty$, because $\xi(n)<\infty$ for each $n$. In summary, although $n_{j}$ does tend to in£nity, it does so rather slowly — too slowly, in fact, for SBPI to converge to the optimal policy. Indeed, $P\left(d_{j}=\alpha\right.$ for all $j \in$ $[\theta(n-1)+1, \theta(n)])<\epsilon$. Consequently for any $k$, we have $P\left(d_{j}=\alpha\right.$ for all $\left.j \geq k\right) \leq \lim _{m \rightarrow \infty} \epsilon^{m}=0$. Therefore, SBPI fails to converge in probability to $\alpha$, and hence does not converge almost surely to $\alpha$. Note also that the specifc construction of the simulation estimators above was chosen to simplify calculations, and is not required for convergence to fail.

So, simulation-based policy iteration need not converge to an optimal policy, even when using unbiased estimates for the solution to the AEE, and letting the number of replications grow to infnity. As we shall see in the coming sections, it is important to ensure that the number of replications grows "fast enough."

\section{The Convergence Result}

In this section, we present our main convergence results concerning the simulation-based policy iteration algorithm. We will provide easily-veri£able conditions under which the SBPI algorithm is, with probability one, absorbed into the set of optimal decision rules. As was shown in Section 3, it does not suffce to simply let the number of simulation replications grow to in£nity.

Before we proceed, we need to brieay describe how SBPI generates simulation estimates. We assume that each estimator $h_{d}^{n}(x)$ is of the form $h_{d}^{n}(x)=\zeta^{n}\left(x, d, U^{n}\right)$, where $U^{n}$ is a random element from a space $\mathbf{U}^{\mathbf{n}}$, and $\zeta^{n}(x, \cdot, \cdot)$ is a deterministic function that maps elements of $D$ and $\mathbf{U}^{\mathbf{n}}$ to realizations of $h_{d}^{n}(x)$. Recall that $\left\{n_{j}\right\}$ is a pre-specifed sequence of parameters in the SBPI algorithm. The quantity $n_{j}$ tells us to use function $\zeta^{n_{j}}$ and space $\mathbf{U}^{\mathbf{n}_{\mathbf{j}}}$ to generate the simulation estimates of a solution of the AEE for the decision rule $d_{j}$. Note that $d_{j}$ is itself random, since it depends upon the outcomes of previous simulations. In Theorem 4.2 below, we assume that $\left\{U^{n_{j}}\right\}$ are independent. This means that conditional upon $d_{j}$, the estimates $h_{d_{j}}^{n_{j}}(x)=\zeta^{n_{j}}\left(x, d_{j}, U^{n_{j}}\right)$ are independent of the prior evolution of SBPI up to iteration $j$. Consequently, 
$d_{j+1}$ is also independent of the prior evolution of SBPI up to iteration $j$, conditional upon $d_{j}$.

One of the important concepts for our general convergence results is the comparison of SBPI with standard deterministic policy iteration. For each decision rule $d \in D$, de£ne

$$
\phi(d) \equiv \arg \max _{d^{\prime} \in D}\left\{r_{d^{\prime}}+P_{d^{\prime}} h_{d}\right\}
$$

i.e., $\phi(d)$ is the set of policies that could be selected in the (standard) policy iteration algorithm when the current policy is $d$.

A key quantity is the probability

$$
q(n, d)=P\left(\arg \max _{d^{\prime} \in D}\left\{r_{d^{\prime}}+P_{d^{\prime}} h_{d}^{n}\right\} \subseteq \phi(d)\right)
$$

The expression (4.1) gives the probability that an optimization using $h_{d}^{n}$ as a proxy for $h_{d}$ yields solutions that are also obtained from an optimization using $h_{d}$. We begin with a well-known lemma; see, for example, [4, Theorem 1.9, p. 422] or [18, Lemma 2.9.1].

Lemma 4.1 If $\sum_{j=0}^{\infty}\left[1-y_{j}\right]<\infty$ and $0 \leq y_{j} \leq 1$ for all $j$, then $\lim _{k \rightarrow \infty} \prod_{j \geq k} y_{j}=1$.

We are now ready to give suffcient conditions for the SBPI algorithm to reach $D^{*}$ and remain there indefnitely with probability one. In Section 5, we will show how the conditions can be applied in various situations. The following is the main result of this section.

Theorem 4.2 Consider the SBPI Algorithm, and suppose that

1. $\left\{U^{n_{j}}: j \geq 0\right\}$ are independent,

2. $\left\{q_{j}: j \geq 0\right\}$ satisfy

$$
\sum_{j=0}^{\infty}\left[1-q_{j}\right]<\infty
$$

where $q_{j} \equiv \min _{d \in D} q\left(n_{j}, d\right)$.

Then there exists a random variable $\ell$ such that

$$
P\left(d_{j} \in D^{*} \text { for all } j \geq \ell\right)=1 \text {. }
$$


Proof. For each $n$, de£ne the event $A_{n}=\left\{d_{j} \in D^{*}\right.$ for all $\left.j \geq n\right\}$. Then, we see that $\left\{\exists \ell: d_{j} \in\right.$ $D^{*}$ for all $\left.j \geq \ell\right\}=\bigcup_{n \geq 0} A_{n}$. Hence,

$$
P\left(\exists \ell: d_{j} \in D^{*} \text { for all } j \geq \ell\right)=P\left(\bigcup_{n \geq 0} A_{n}\right)=\lim _{n \rightarrow \infty} P\left(A_{n}\right)
$$

Therefore, it suffces to show that $\lim _{n \rightarrow \infty} P\left(A_{n}\right)=1$.

Let $\tilde{n}(d)$ be the maximum number of steps required for the deterministic policy iteration algorithm, starting from decision rule $d$, to reach an optimal decision rule, and let $\tilde{n}=\sup _{d} \tilde{n}(d)$. Since we have assumed our problem to be unichain with £nite state and action spaces, it follows from [17, Theorem 8.6.6, p. 383] that $\tilde{n}<\infty$. Also by virtue of [17, Proof of Theorem 8.6.6, p. 383], we see that if the sequence of decision rules produced by the SBPI algorithm, $d_{0}, d_{1}, \ldots, d_{\tilde{n}}$, satisfy $d_{j} \in \phi\left(d_{j-1}\right)$ for $j=1, \ldots, \tilde{n}$, then $d_{\tilde{n}}$ is an optimal policy. Similarly, if $d \in D^{*}$, then $\phi(d) \subseteq D^{*}$. The key fact is that once deterministic policy iteration arrives at an average-reward optimal policy, running further iterations cannot cause it to move to a suboptimal policy. Therefore, for $n \geq \tilde{n}$, we have that

$$
A_{n} \supseteq\left\{d_{j} \in \phi\left(d_{j-1}\right) \text { for all } j \geq n\right\} \supseteq\left\{d_{j} \in \phi\left(d_{j-1}\right) \text { for all } j>n-\tilde{n}\right\},
$$

and so

$$
P\left(A_{n}\right) \geq P\left(d_{j} \in \phi\left(d_{j-1}\right) \text { for all } j>n-\tilde{n}\right) \geq \prod_{j>n-\tilde{n}} q_{j-1}
$$

The second inequality above follows from the independence of the sequence $\left\{U^{n_{j}}\right\}$. Assumption (4.2) and Lemma 4.1 together imply that $\lim _{n \rightarrow \infty} \prod_{j>n-\tilde{n}} q_{j-1}=1$. Thus, $P\left(A_{n}\right) \rightarrow 1$ as $n \rightarrow \infty$, which completes the proof.

The independence assumption on the sequence $\left\{U^{n_{j}}\right\}$ ensures that $\left\{d_{j}\right\}$ forms a (non-homogeneous) Markov chain on $D$. This basic structure would be preserved if we also used $\left\{U^{n_{j}}\right\}$ to estimate the transition probabilities that are required for the policy improvement step. If we did this, and redefned $q(n, d)$ to be simply the probability of getting a "correct" maximizer, then Theorem 4.2 could also be applied to problems with unknown transition probabilities. However, we shall not venture further down this path.

\section{Simulation Estimates for Solutions to the Average Evaluation Equations}

Theorem 4.2 gives conditions under which SBPI eventually hits, and never leaves, the set of optimal policies. Condition (4.2) is the key to that result, but it is not a natural condition to check in applications. In this 
section we discuss three different estimators of solutions to the AEE, and in each case, give easily veri£able conditions that, in turn, imply (4.2).

We frst give some preliminary results that streamline the presentation. For a given policy $d^{\infty}$, recall that the AEE have multiple solutions, all of which differ by an additive constant term. Let $h_{d}$ be a solution to the AEE, and let $h_{d}^{n}$ be an estimator of $h_{d}$, where $n$ is related to the simulation runlength. The specifc de£nition of $h_{d}$ and $h_{d}^{n}$ will vary. Let $n_{j}$ denote the runlength used to estimate $h_{d_{j}}$ on the $j$ th iteration of SBPI, where $d_{j}^{\infty}$ is the policy at the $j$ th iteration. For a function $v: \mathbb{X} \rightarrow \mathbb{R}$, de£ne $\|v\|=\max _{x \in \mathbb{X}}|v(x)|$.

Lemma 5.1 There exists $\delta>0$ such that if

$$
\sum_{j=0}^{\infty} \max _{d \in D} P\left(\left\|h_{d}^{n_{j}}-h_{d}\right\|>\delta\right)<\infty
$$

then (4.2) holds.

Proof. To verify (4.2), observe that for a given policy $d^{\infty}$, there exists $\delta_{d}>0$ such that for any $h$ that satis£es $\left\|h-h_{d}\right\|<\delta_{d}$

$$
\arg \max _{a \in A_{x}}\left\{r(x, a)+\sum_{y \in \mathbb{X}} p(y \mid x, a) h(y)\right\} \subseteq \arg \max _{a \in A_{x}}\left\{r(x, a)+\sum_{y \in \mathbb{X}} p(y \mid x, a) h_{d}(y)\right\} \quad \text { for all } x \in \mathbb{X} .
$$

If we take $\delta=\min _{d} \delta_{d}$ (recall that there are only a £nite number of stationary deterministic policies, so that $\delta>0)$ then $q(n, d) \geq P\left(\left\|h_{d}^{n}-h_{d}\right\| \leq \delta\right)$. The result now follows.

We immediately obtain the following corollary. Let $\operatorname{mse}(X)$ denote the mean squared error $E(X-\mu)^{2}$ of the estimator $X$ of $\mu$.

Corollary 5.2 Suppose that for all $x \in \mathbb{X}$ and $d^{\infty} \in D^{\infty}$, $\operatorname{mse}\left(h_{d}^{n_{j}}(x)\right)=E\left(h_{d}^{n_{j}}(x)-h_{d}(x)\right)^{2} \leq c_{d} / n_{j}$ for some deterministic constant $c_{d}$. If

$$
\sum_{j=1}^{\infty} \frac{1}{n_{j}}<\infty
$$

then (4.2) holds. 
Proof. Let $\delta$ be as in Lemma 5.1. By Boole's and Chebychev's inequalities

$$
\begin{aligned}
P\left(\left\|h_{d}^{n_{j}}-h_{d}\right\|>\delta\right) & =P\left(\bigcup_{x \in \mathbb{X}}\left\{\left|h_{d}^{n_{j}}(x)-h_{d}(x)\right|>\delta\right\}\right) \\
& \leq \sum_{x \in \mathbb{X}} P\left(\left|h_{d}^{n_{j}}(x)-h_{d}(x)\right|>\delta\right) \\
& \leq \sum_{x \in \mathbb{X}} \frac{\operatorname{mse}\left(h_{d}^{n_{j}}(x)\right)}{\delta^{2}} \\
& \leq \sum_{x \in \mathbb{X}} \frac{c_{d}}{n_{j} \delta^{2}} .
\end{aligned}
$$

Since $D^{\infty}$ is £nite, the result follows by Lemma 5.1.

We now apply these results to three different estimators of a solution to the AEE. In what follows, we let $X=\left\{X_{i}: i \geq 0\right\}$ denote the Markov chain induced by a policy $d^{\infty} \in D^{\infty}$. To maintain readability, we suppress the dependence of $X$ on $d$.

\subsection{An Unbiased Estimate of the Bias}

Defne $h$ to be the bias,

$$
h(x)=\sum_{i=0}^{\infty} E^{x}\left[r\left(X_{i}\right)-g\right],
$$

where $E^{x}$ denotes expectation with respect to the probability measure under which $X_{0}=x$. As has previously been discussed, $(g, h)$ solve the AEE. We can estimate $h$ using simulation as follows.

Suppose that it is possible to obtain samples from the stationary distribution, $\pi$ say, of the Markov chain $\left\{X_{i}: i \geq 0\right\}$. Let $Y=\left\{Y_{i}: i \geq 0\right\}$ denote a version of the chain with initial state $Y_{0}$ sampled from $\pi$, so that $Y$ is stationary. Similarly, let $X^{x}=\left\{X_{i}^{x}: i \geq 0\right\}$ denote a version of the chain initiated in state $x$. We require that $X^{x}$ and $Y$ be constructed on a common probability space. De£ne the stopping time

$$
\eta=\inf \left\{k \geq 0: X_{k}^{x}=Y_{k}\right\}
$$

to be the £rst time that the two sample paths meet. To ensure that $\eta$ is £nite, we will typically require, in addition to the unichain assumption, that the chain be aperiodic. We constrain the joint construction so that for $k>\eta, X_{k}^{x}=Y_{k}$; i.e., after the two processes meet, they "stick" together. 
Now, let

$$
H(x)=\sum_{k=0}^{\eta}\left[r\left(X_{k}^{x}\right)-r\left(Y_{k}\right)\right]
$$

be the difference in rewards accrued between the two chains until they meet at time $\eta$. Under appropriate conditions, $H(x)$ is an unbiased estimator for the bias $h(x)$, as the following result shows.

Proposition 5.3 Suppose that $d$ is a stationary deterministic policy and that $E \eta<\infty$, where E denotes expectation with respect to the probability measure on the space on which both $X^{x}$ and $Y$ are constructed. Then $E H(x)=h(x)$.

Proof. We have that

$$
\begin{aligned}
E H(x) & =E \sum_{k=0}^{\infty}\left[r\left(X_{k}^{x}\right)-r\left(Y_{k}\right)\right] I(\eta \geq k) \\
& =\sum_{k=0}^{\infty} E\left[r\left(X_{k}^{x}\right)-r\left(Y_{k}\right)\right] I(\eta \geq k) \\
& =\sum_{k=0}^{\infty} E\left[r\left(X_{k}^{x}\right)-r\left(Y_{k}\right)\right]-\sum_{k=0}^{\infty} E\left[r\left(X_{k}^{x}\right)-r\left(Y_{k}\right)\right] I(\eta<k),
\end{aligned}
$$

where the interchange in (5.5) is justifed since the rewards $r(\cdot)$ are bounded and $E \eta<\infty$. The frst term in (5.6) is the bias as given by $h(x)$, since $\operatorname{Er}\left(Y_{k}\right)=g$. The second term in (5.6) is 0 , because $X_{k}^{x}=Y_{k}$ for $k>\eta$.

Proposition 5.3 gives conditions under which we can construct a random variable $H(x)$ whose expectation is $h(x)$. By repeating the above construction $n$ independent times to yield $H^{1}(x), H^{2}(x), \ldots, H^{n}(x)$, we can obtain an unbiased estimator $h^{n}(x)$ of $h(x)$ given by

$$
h^{n}(x)=\frac{1}{n} \sum_{i=1}^{n} H^{i}(x) .
$$

A critical ingredient in constructing the estimator $h^{n}(x)$ is obtaining a sample from $\pi$ to serve as the initial point in a sample path of $Y$. Such samples can be obtained through the use of "perfect sampling" 
for Markov chains, of which the most widely used method is currently "coupling from the past" $[15,16]$. Unlike the approach of gathering samples after simulating the chain for some predetermined "burn-in" time, the samples are exactly distributed according to $\pi$. Furthermore, by repeating the coupling-from-thepast construction several independent times, we can obtain independent samples from $\pi$. An accessible introduction to coupling from the past is given by [23].

It is well-known that it is possible to construct $X^{x}$ and $Y$ so that their forward coupling time (5.4) has a £nite moment generating function in a neighborhood of the origin; see, e.g., [19, p. 419] or [20, p. 45]. This implies, in particular, that

$$
E\left[\left(\eta_{d}(x)\right)^{2}\right]<\infty \quad \forall x \in \mathbb{X},
$$

where we have explicitly included the dependence of the coupling time $\eta$ on the policy $d^{\infty}$ and the starting state $x$ of $\left\{X_{k}^{x}\right\}$.

Lemma 5.4 Suppose that (5.7) holds for all $d^{\infty} \in D^{\infty}$. Then there exists a constant $\kappa<\infty$ such that

$$
\sup _{x \in \mathbb{X}, d \in D} \operatorname{var}\left(H_{d}(x)\right)<\kappa
$$

Proof. For any state $x \in \mathbb{X}$ and policy $d^{\infty} \in D^{\infty}$,

$$
E\left[\left(H_{d}(x)\right)^{2}\right] \leq E\left(\sum_{k=0}^{\eta_{d}(x)-1} 2\left\|r_{d}\right\|\right)^{2}=4\left\|r_{d}\right\|^{2} E\left[\left(\eta_{d}(x)\right)^{2}\right]<\infty
$$

The result now follows from the assumption that the state and action spaces are £nite.

We can now apply Corollary 5.2 to obtain the following result. The proof is immediate from Lemma 5.4 and the unbiasedness of the bias estimator $h^{n}$, since $\operatorname{mse}\left(h^{n}(x)\right)=\operatorname{var} H(x) / n$ for all $x \in \mathbb{X}$.

Proposition 5.5 Suppose that (5.7) holds. If

$$
\sum_{j=0}^{\infty} \frac{1}{n_{j}}<\infty
$$

then (4.2) holds. 
In concert with Theorem 4.2, Proposition 5.5 gives easily veri£ed suffcient conditions for SBPI to converge almost surely to an optimal policy. The condition (5.2) basically states that the runlengths must grow "fast enough" that we eventually do not erroneously step out of the set of optimal policies. Note that this condition is not satis£ed by taking $n_{j}$ to be constant, no matter how large, i.e., "£xed runlengths are not enough."

The assumption that the forward coupling time possesses a fnite moment generating function in a neighborhood of the origin allows us to weaken the condition (5.2). In particular, if for every $x$ and $d$ there exists $\theta_{x, d}>0$ so that

$$
E e^{\theta H_{d}(x)}<\infty \quad \text { for }|\theta|<\theta_{x, d},
$$

then a standard large deviations result, e.g., [9, Theorem 6, p. 281], ensures that for any $\bar{\delta}>0$, there exists $0<\gamma_{x, d}<1$ so that $P\left(\left|h_{d}^{n}(x)-h_{d}(x)\right|>\bar{\delta}\right) \leq 2 \gamma_{x, d}^{n}$. Provided the state and action spaces are £nite, we can modify the derivation of (5.3) to conclude that there exist constants $c>0$ and $0<\gamma<1$ so that

$$
P\left(\left\|h_{d}^{n}-h_{d}\right\|>\bar{\delta}\right)<c \gamma^{n}
$$

Combining this with Lemma 5.1, we obtain the following result.

Proposition 5.6 Suppose that (5.9) holds. If $\gamma$ satis£es (5.10) and

$$
\sum_{j=0}^{\infty} \gamma^{n_{j}}<\infty
$$

then (4.2) holds.

It is unlikely that one will be able to identify $\gamma$ satisfying (5.10) a priori, but observe that we could also employ the stronger condition that (5.11) holds with $\gamma=\nu$ for all $\nu \in(0,1)$, which is a condition that does not rely on a priori knowledge. For example, if $n_{j}=j$, then this condition holds, but (5.2) does not. In this case, stronger moment assumptions do indeed yield a "payoff."

\subsection{An Estimate of the Relative Value Function}

The estimator of the bias constructed in the previous section relies on obtaining a sample from the stationary distribution of the chain. Perfect sampling may be employed for any unichain, aperiodic, £nite state space 
Markov chain to obtain such samples. However, the computational effort required to perform perfect sampling can grow exorbitantly as the state space increases in size. Motivated by this observation, we show how to construct a simulation estimator of a different solution to the AEE.

Let $x^{*} \in \mathbb{X}$ be a recurrent state, and let $\tau=\inf \left\{n \geq 0: X_{n}=x^{*}\right\}$ denote the hitting time of $x^{*}$. A solution to the AEE is then given by the relative value function

$$
h(x)=E^{x} \sum_{i=0}^{\tau-1}\left[r\left(X_{i}\right)-g\right] .
$$

(Again, we are suppressing dependence on the policy $d$ in our notation. Note also that we are rede£ning $h$ within this section.) We can construct an estimator of $h(x)$ as follows.

The gain $g$ can be estimated via

$$
g^{n}=\frac{1}{n} \sum_{i=0}^{n-1} r\left(X_{i}\right)
$$

with an arbitrary initial distribution on the chain. Once this point estimate is obtained, for all $x \in \mathbb{X}, h(x)$ may be estimated by $h^{m, n}(x)$, where $h^{m, n}(x)$ is the sample mean of $m$ conditionally independent (given $\left.g^{n}\right)$ replicates of

$$
H^{n}(x)=\sum_{j=0}^{\tau-1}\left[r\left(X_{j}^{x}\right)-g^{n}\right] .
$$

Remark 5.7 The estimator $h^{m, n}$ of $h$ is typically biased because of the presence of bias in the estimator $g^{n}$ of $g$. If $g^{n}$ is an unbiased estimator of $g$, then $h^{m, n}$ is an unbiased estimator of the relative value function $h$ (see also $[13,12])$.

We then obtain the following result on convergence of SBPI. Let $m_{j}$ and $n_{j}$ represent the runlengths used to estimate $h=h_{d_{j}}$ on the $j$ th iteration of SBPI. For the following result we need not use the same "reference" state $x^{*}$ at each iteration of SBPI; we require only that the reference state used to construct the estimator of $h_{d_{j}}$ be a recurrent state for decision rule $d_{j}$.

\section{Proposition 5.8 If}

$$
\sum_{j=0}^{\infty} \max \left\{\frac{1}{n_{j}}, \frac{1}{m_{j}}\right\}<\infty
$$

and the estimator $h^{m, n}$ of $h$ outlined above is used in SBPI, then (4.2) holds. 
Proof. We will show that the mean squared error of $h^{m, n}(x)$ is of the order $\max \left\{m^{-1}, n^{-1}\right\}$ so that the result will follow immediately from Corollary 5.2. We append the suf£x $(i)$ to quantities to indicate that they relate to the $i$ th independent replication, so that $\tau(i)$ is the time to hit $x^{*}$ in the $i$ th independent replication of $H^{n}$, etc. We then have that

$$
\begin{aligned}
\operatorname{mse}\left(h^{m, n}(x)\right) & =E\left[h^{m, n}(x)-h(x)\right]^{2} \\
& =E\left[\frac{1}{m} \sum_{i=1}^{m}\left(\sum_{j=0}^{\tau(i)-1}\left[r\left(X_{j}^{x}(i)\right)-g\right]+\sum_{j=0}^{\tau(i)-1}\left(g-g^{n}\right)\right)-h(x)\right]^{2} \\
& =E\left[\frac{1}{m}\left(\sum_{i=1}^{m} D_{i}(x)\right)+\left(g-g^{n}\right) \bar{\tau}(m)\right]^{2} \\
& \leq 2 E\left[\frac{1}{m} \sum_{i=1}^{m} D_{i}(x)\right]^{2}+2 E\left[\left(g-g^{n}\right)^{2} \bar{\tau}(m)^{2}\right]
\end{aligned}
$$

where $D_{i}(x)=\sum_{j=0}^{\tau(i)-1}\left[r\left(X_{j}^{x}(i)\right)-g\right]-h(x)$, and $\bar{\tau}(m)$ is the sample mean of $\tau(i), i=1, \ldots, m$. Equation (5.12) follows since $(a+b)^{2} \leq 2 a^{2}+2 b^{2}$ for any real numbers $a$ and $b$.

Now, because of the unichain and £nite state space assumptions, $\tau$ has a moment generating function and hence moments of all orders under $E^{x}$ for any $x \in \mathbb{X}$. In particular, $E^{x} \tau^{2}<\infty$ for all $x \in \mathbb{X}$. It then follows, as in Lemma 5.4, that $E^{x} D_{1}(x)^{2}<\infty$ since $r$ is bounded. Since $E^{x} D_{1}(x)=0$, the frst term in (5.12) equals $2 E^{x} D_{1}(x)^{2} / m$.

Turning to the second term above, $\bar{\tau}(m)$ is independent of $g^{n}$, so that the second term in (5.12) is equal to $2 \mathrm{mse}\left(g^{n}\right) E^{x} \bar{\tau}_{m}^{2}$. In addition,

$$
E^{x} \bar{\tau}_{m}^{2}=\frac{E^{x} \tau^{2}}{m}+\frac{m(m-1)\left(E^{x} \tau\right)^{2}}{m^{2}}
$$

and $E^{x} \tau^{2}<\infty$, so that $E^{x} \bar{\tau}_{m}^{2}$ is bounded. Therefore, the proof will be complete once we show that mse $\left(g^{n}\right) \leq c / n$ for some $c>0$. Let $\mu$ denote the initial distribution on the chain when computing $g^{n}$. Theorem 6.5 of [4] gives that $\operatorname{var}_{\mu}\left(g^{n}\right) \leq c^{\prime} / n$ for some $c^{\prime}>0$ (for any initial distribution), so it remains to establish that the bias of $g^{n}, E^{\mu} g^{n}-g \leq c^{\prime \prime} / \sqrt{n}$, where $E^{\mu}$ denotes the expectation operator on the path 
space of the chain with initial distribution $\mu$. Let

$$
b(x)=\sum_{n=0}^{\infty} E^{x}\left[r\left(X_{n}\right)-g\right],
$$

which is $£$ nite for all $x \in \mathbb{X}$, since $\mathbb{X}$ is $£$ nite and the unichain property is in effect. Now observe that

$$
\begin{aligned}
E^{\mu} g^{n}-g & =\sum_{x \in \mathbb{X}} \mu(\{x\}) E^{x} \frac{1}{n} \sum_{i=0}^{n-1}\left[r\left(X_{i}\right)-g\right] \\
& =\sum_{x \in \mathbb{X}} \mu(\{x\}) \frac{1}{n} \sum_{i=0}^{n-1} E^{x}\left[r\left(X_{i}\right)-g\right] \\
& =\sum_{x \in \mathbb{X}} \mu(\{x\})\left[\frac{1}{n} \sum_{i=0}^{\infty} E^{x}\left[r\left(X_{i}\right)-g\right]-\frac{1}{n} \sum_{i=n}^{\infty} E^{x}\left[r\left(X_{i}\right)-g\right]\right] \\
& =\sum_{x \in \mathbb{X}} \mu(\{x\})\left[\frac{1}{n} b(x)+o\left(n^{-1}\right)\right] \\
& =\frac{1}{n} \sum_{x \in \mathbb{X}} \mu(\{x\}) b(x)+o\left(n^{-1}\right),
\end{aligned}
$$

where $o\left(a_{n}\right)$ denotes a sequence $\left\{w_{n}\right\}$ with the property that $w_{n} / a_{n} \rightarrow 0$ as $n \rightarrow \infty$. Thus, the bias in $g^{n}$ is bounded by $c^{\prime \prime} / n$ for some $c^{\prime \prime}$, and the proof is complete.

\subsection{A Ratio Estimator}

The estimator constructed in the previous section relies on a preliminary simulation to estimate the gain $g$. One might prefer an estimator that can be obtained from a single simulation run, and in this section we consider such an estimator. The estimator constructed here relies on the chain visiting each $x \in \mathbb{X}$ infnitely often. The unichain assumption that has previously been in effect is too weak, since some states may be transient. Therefore, the estimator constructed here only applies when all chains arising from stationary, deterministic policies $d^{\infty}$ are irreducible. Note that this is equivalent to all stationary, deterministic policies yielding a single common recurrent class and no transient states.

Let $T(0)=0$ and for $n \geq 0$ de£ne $T(n+1)=\inf \left\{k>T(n): X_{k}=x^{*}\right\}$ to be the consecutive hitting times of the distinguished recurrent state $x^{*} \in \mathbb{X}$. Suppose that $X_{0}=x^{*}$, so that the times $T(0), T(1), \ldots$ divide the sample path of $X=\left\{X_{k}: k \geq 0\right\}$ into i.i.d. regenerative cycles. 
Let

$$
g^{n}=\frac{\sum_{i=0}^{T(n)-1} r\left(X_{k}\right)}{T(n)}
$$

be an estimate of the gain based on $n$ regenerative cycles. For $i \geq 1$ de£ne

$$
\begin{aligned}
W_{i}(x) & =\sum_{j=T(i-1)}^{T(i)-1}\left(r\left(X_{j}\right)-g^{n}\right) N_{x}(j, i), \text { and } \\
C_{i}(x) & =\sum_{j=T(i-1)}^{T(i)-1} I\left(X_{j}=x\right),
\end{aligned}
$$

where

$$
N_{x}(j, i)=\sum_{k=T(i-1)}^{j} I\left(X_{k}=x\right)
$$

is the number of visits to state $x$ by time $j$ within the $i$ th regenerative cycle. Here, $C_{i}(x)$ gives the number of visits to state $x$ in the $i$ th regenerative cycle. The expression for $W_{i}(x)$ will be discussed further below.

Defne the estimator $h^{n}(x)$ by

$$
h^{n}(x)=\frac{\bar{W}_{n}(x)}{\bar{C}_{n}(x)}
$$

if $\bar{C}_{n}(x)>0$, and 1 otherwise, where $\bar{W}_{n}(x)$ and $\bar{C}_{n}(x)$ denote sample means of $W_{1}(x), \ldots, W_{n}(x)$ and $C_{1}(x), \ldots, C_{n}(x)$ respectively. In contrast to previous sections where $n$ represented the simulation runlength in terms of number of transitions, here $n$ represents the number of completed regenerative cycles.

Here $h^{n}(x)$ is used as an estimator of $h(x)$, where

$$
h(x)=E^{x} \sum_{k=0}^{\tau-1}\left[r\left(X_{k}\right)-g\right]
$$

is the expected cumulative cost (centered by the gain $g$ ) until hitting the state $x^{*}$ starting from state $x$. (Recall that $\tau=\inf \left\{k \geq 0: X_{k}=x^{*}\right\}$ is the hitting time of state $x^{*}$.) 
To see why $h^{n}$ is a reasonable estimator of $h$, note that

$$
\begin{aligned}
W_{i}(x) & =\sum_{j=T(i-1)}^{T(i)-1}\left(r\left(X_{j}\right)-g^{n}\right) \sum_{k=T(i-1)}^{j} I\left(X_{k}=x\right) \\
& =\sum_{k=T(i-1)}^{T(i)-1} I\left(X_{k}=x\right) \sum_{j=k}^{T(i)-1}\left(r\left(X_{j}\right)-g^{n}\right)
\end{aligned}
$$

so that $W_{i}(x)$ is a sum of terms, each starting in state $x$ and representing the cumulative centered cost until the end of the current regenerative cycle when the chain hits state $x^{*}$. Thus $W_{i}(x)$ is a sum of (dependent) terms, each of which is a reasonable estimator of $h(x)$. The estimator given by Equation (18) in [6] is similar to $h^{n}$ as defned above, but it only counts the £rst time (if any) in each regenerative cycle that the chain visits state $x$. The following proposition establishes that $h^{n}$ has mean squared error of order $n^{-1}$.

Proposition 5.9 Suppose that the chain $X=\left\{X_{n}: n \geq 0\right\}$ is irreducible. Then the mse $\left(h^{n}(x)\right) \leq c n^{-1}$ for some $c<\infty$. Hence, if all stationary deterministic decision rules give rise to irreducible chains, and $n_{j}$ denotes the number of regenerative cycles used in SBPI iteration $j$, then

$$
\sum_{j=0}^{\infty} \frac{1}{n_{j}}<\infty
$$

implies that (4.2) holds.

Proof. We only need to establish the mean squared error result, since an application of Corollary 5.2 then completes the proof.

Let us $£ x \in \mathbb{X}$ and then drop the dependence in our notation on $x$, so that $C_{i}=C_{i}(x)$ etc. For $i \geq 1$, defne $\eta_{i}=T(i)-T(i-1)$ to be the length of the $i$ th regenerative cycle, and note that $\eta_{i}$ represents the hitting time of state $x^{*}$ in a £nite state space irreducible Markov chain. Therefore, $\eta_{i}$ has a £nite moment generating function in a neighborhood of the origin, $i \geq 1$. Since $0 \leq C_{i} \leq \eta_{i}, C_{i}$ also has a £nite moment generating function in a neighborhood of the origin, $i \geq 1$. Furthermore, if we defne the i.i.d. sequence $\left\{V_{i}: i \geq 1\right\}$ by

$$
V_{i}=W_{i}+\left(g^{n}-g\right) \sum_{j=T(i-1)}^{T(i)-1} N_{x}(j, i)=\sum_{j=T(i-1)}^{T(i)-1}\left[r\left(X_{j}\right)-g\right] N_{x}(j, i),
$$


then since $\left|V_{i}\right| \leq 2\|r\| \eta_{i}^{2}, V_{i}$ also has a Enite moment generating function in a neighborhood of the origin.

For notational convenience, defne

$$
D_{i}=\sum_{j=T(i-1)}^{T(i)-1} N_{x}(j, i)
$$

Note that

$$
n E\left(h^{n}-h\right)^{2}=n E\left[\frac{\bar{W}_{n}}{\bar{C}_{n}}-\frac{E V_{1}}{E C_{1}}\right]^{2} I\left(\bar{C}_{n}>0\right)+n(1-h)^{2} P\left(\bar{C}_{n}=0\right) .
$$

Now, the chain is irreducible and recurrent, so that all states are visited infnitely often. In particular, then, $E C_{1}>0$ and $P\left(C_{1}=0\right)<1$. It follows that $n(1-h)^{2} P\left(\bar{C}_{n}=0\right)=n(1-h)^{2} P\left(C_{1}=0\right)^{n} \rightarrow 0$ as $n \rightarrow \infty$ and is therefore $o(1)$. To establish the result, it remains to show that the frst term in (5.13) is bounded.

The identity $(a+b+c)^{2} \leq 3 a^{2}+3 b^{2}+3 c^{2}$ allows us to conclude that

$$
\begin{aligned}
n E\left(h^{n}-h\right)^{2}= & n E\left[\frac{\bar{V}_{n}}{\bar{C}_{n}}+\left(g-g^{n}\right) \frac{\bar{D}_{n}}{\bar{C}_{n}}-\frac{E V_{1}}{E C_{1}}\right]^{2} I\left(\bar{C}_{n}>0\right)+o(1) \\
= & n E\left[\frac{\bar{V}_{n}-E V_{1}}{\bar{C}_{n}}+E V_{1}\left(\frac{1}{\bar{C}_{n}}-\frac{1}{E C_{1}}\right)+\left(g-g^{n}\right) \frac{\bar{D}_{n}}{\bar{C}_{n}}\right]^{2} I\left(\bar{C}_{n}>0\right)+o(1) \\
\leq & 3 n E\left[\frac{\bar{V}_{n}-E V_{1}}{\bar{C}_{n}}\right]^{2} I\left(\bar{C}_{n}>0\right)+3 n\left(E V_{1}\right)^{2} E\left[\frac{1}{\bar{C}_{n}}-\frac{1}{E C_{1}}\right]^{2} I\left(\bar{C}_{n}>0\right) \\
& +3 n E\left[\left(g-g^{n}\right) \frac{\bar{D}_{n}}{\bar{C}_{n}}\right]^{2} I\left(\bar{C}_{n}>0\right)+o(1) .
\end{aligned}
$$

The remainder of the proof consists of showing that each of the terms in (5.14) are bounded in $n$. 
Note that

$$
\begin{aligned}
& 3 n E\left[\frac{\bar{V}_{n}-E V_{1}}{\bar{C}_{n}}\right]^{2} I\left(\bar{C}_{n}>0\right) \\
& \quad=3 n E\left[\frac{\bar{V}_{n}-E V_{1}}{\bar{C}_{n}}\right]^{2} I\left(\bar{C}_{n} \in\left(0, E C_{1} / 2\right]\right)+3 n E\left[\frac{\bar{V}_{n}-E V_{1}}{\bar{C}_{n}}\right]^{2} I\left(\bar{C}_{n}>E C_{1} / 2\right) \\
& \leq 3 n^{3} E\left[\left(\bar{V}_{n}-E V_{1}\right)^{2} I\left(\bar{C}_{n} \in\left(0, E C_{1} / 2\right]\right)\right]+\frac{12 n}{\left(E C_{1}\right)^{2}} E\left[\left(\bar{V}_{n}-E V_{1}\right)^{2} I\left(\bar{C}_{n}>E C_{1} / 2\right)\right] \\
& \leq 3 n^{3} E\left[\left(\bar{V}_{n}-E V_{1}\right)^{4}\right]^{1 / 2} P\left(\bar{C}_{n} \in\left(0, E C_{1} / 2\right]\right)^{1 / 2}+\frac{12 n}{\left(E C_{1}\right)^{2}} E\left(\bar{V}_{n}-E V_{1}\right)^{2} \\
& \quad=3 E\left[\left(\bar{V}_{n}-E V_{1}\right)^{4}\right]^{1 / 2} n^{3} P\left(\bar{C}_{n} \in\left(0, E C_{1} / 2\right]\right)^{1 / 2}+\frac{12 \operatorname{var} V_{1}}{\left(E C_{1}\right)^{2}} .
\end{aligned}
$$

The £rst inequality follows since $\bar{C}_{n} \geq n^{-1}$ on the event $\bar{C}_{n} \in\left(0, E C_{1} / 2\right]$. To see why, note that $\bar{C}_{n}>0$ implies that $C_{i}>0$ for at least one $i$, and $C_{i}$ is integer valued. The second inequality is a consequence of the Cauchy-Schwarz inequality. Finally, by direct calculation, we can show that $E\left[\left(\bar{V}_{n}-E V_{1}\right)^{4}\right]$ is bounded in $n$ (in fact, it is of the order $\left.n^{-2}\right)$, and since $P\left(\bar{C}_{n} \in\left(0, E C_{1} / 2\right]\right)$ converges to 0 exponentially fast, $n^{3} P\left(\bar{C}_{n} \in\left(0, E C_{1} / 2\right]\right)$ is also bounded in $n$. Thus, we have shown that the frst term in (5.14) is bounded in $n$. A similar approach can be used to show that the remaining terms in (5.14) are also bounded in $n$, and so the proof is complete.

\section{Conclusions}

We have analyzed the convergence of simulation-based policy iteration for average-reward Markov decision processes with £nite state and action spaces. By way of an example, we have shown that allowing simulation runlengths to grow to in£nity does not, in general, suffce to ensure convergence, even in probability, of SBPI. Arguing from £rst principles, we have derived suf£cient conditions for SBPI to be absorbed into the set of optimal decision rules with probability one. Subsequently, we demonstrated how these general conditions can be applied to three different simulation estimators in order to obtain simple, easily-veri£ed conditions that ensure the desired almost-sure convergence of SBPI.

One might very well ask which of the three estimators of solutions to the AEE given in Section 5 one should use in a given situation. The estimator in Section 5.3 is perhaps the least complex to implement, as 
it can be applied based on a single simulated sample path. It has the disadvantage that it requires that every stationary deterministic policy gives rise to an irreducible chain, which is stronger than the unichain requirement. If this assumption is untenable, then one might turn to the estimators given in Sections 5.1 and 5.2 which only require the unichain property. The estimator in Section 5.1 is desirable in that it produces unbiased estimates of the bias. It has the disadvantage that it requires "perfect sampling capability". If one is unwilling, or unable, to implement such a capability, then the estimator in Section 5.2 might be considered.

\section{Acknowledgments}

The work of the second author was supported in part by National Science Foundation grant number DMI0085165.

\section{References}

[1] A. G. Barto, R. S. Sutton, and C. W. Anderson. Neuron-like elements that can solve diffcult learning control problems. IEEE Transactions on Systems, Man and Cybernetics, 13:835-846, 1983.

[2] Dimitri P. Bertsekas. Dynamic Programming and Optimal Control, Volume II. Athena Scientifc, Belmont, 1995.

[3] Dimitri P. Bertsekas and John N. Tsitsiklis. Neuro-Dynamic Programming. Athena Scientifc, Belmont, 1996.

[4] Pierre Brémaud. Markov Chains: Gibbs Fields, Monte Carlo Simulation, and Queues. Springer, New York, 1999.

[5] Xi-Ren Cao. The relations among potentials, perturbation analysis, and Markov decision processes. Discrete-Event Dynamic Systems: Theory and Applications, 8:71-87, 1998.

[6] X. R. Cao. Single sample path-based optimization of Markov chains. Journal of Optimization Theory and Applications, 100:527-548, 1999.

[7] Xi-Ren Cao. A unifed approach to Markov decision problems and performanace sensitivity analysis. Automatica, 36:771-774, 2000. 
[8] Xi-Ren Cao and Han-Fu Chen. Perturbation realization, potentials, and sensitivity analysis of Markov processes. IEEE Transactions on Automatic Control, 42(10):1382-1393, October 1997.

[9] Bert Fristedt and Lawrence Gray. A Modern Approach to Probability Theory. Birkhauser, Boston, 1997.

[10] Ronald A. Howard. Dynamic Programming and Markov Processes. John Wiley \& Sons, Inc., New York, 1960.

[11] Vijaymohan R. Konda and Vivek S. Borkar. Actor-critic-type learning algorithms for Markov decision processes. SIAM Journal on Control and Optimization, 38:94-123, 1999.

[12] Mark E. Lewis and Martin L. Puterman. Bias optimality. In Eugene Feinberg and Adam Shwartz, editors, Handbook of Markov Decision Processes: Methods and Applications. Kluwer Academic Publishers, 2001. in press.

[13] Mark E. Lewis and Martin L. Puterman. A probabilistic analysis of bias optimality in unichain Markov decision processes. IEEE Transactions on Automatic Control, 46(1):96-100, January 2001.

[14] Sean P. Meyn and Richard L. Tweedie. Markov Chains and Stochastic Stability. Springer-Verlag, London, 1993.

[15] J. G. Propp and D. B. Wilson. Exact sampling with coupled Markov chains and applications to statistical mechanics. Random Structures and Algorithms, 9:223-252, 1996.

[16] J. G. Propp and D. B. Wilson. How to get a perfectly random sample from a generic Markov chain and generate a random spanning tree of a directed graph. Journal of Algorithms, 27:170-217, 1998.

[17] Martin L. Puterman. Markov Decision Processes: Discrete Stochastic Dynamic Programming. John Wiley and Sons, Inc., New York, 1994.

[18] Sidney Resnick. Adventures in Stochastic Processes. Birkhauser, Boston, 1992.

[19] Sheldon M. Ross. Stochastic Processes. John Wiley and Sons, Inc., New York, 2nd edition, 1996.

[20] Hermann Thorisson. Coupling, Stationarity, and Regeneration. Springer-Verlag, New York, 2000. 
[21] J. Tsitsiklis. Asynchronous stochastic approximation and Q-learning. Machine Learning, 16:195-202, 1994.

[22] C. J. C. H. Watkins and P. Dayan. Q-learning. Machine Learning, 8:279-292, 1992.

[23] D. B. Wilson. Layered multishift coupling for use in perfect sampling algorithms (with a primer on CFTP), volume 26 of Fields Institute Communications, pages 141-176. American Mathematical Society, 2000. 